\title{
Research Progress of Geraniol in Tumor Therapy
}

Zhi Hao ${ }^{1 *}$, Jianying $\mathrm{Cui}^{2}$, Hongli Yang ${ }^{1}$, Yonggang Zhang ${ }^{1}$, Meng Zhu ${ }^{1}$

${ }^{1}$ Department of head and neck surgery, Affiliated Hospital of Hebei University, Baoding 071000, Hebei Province, China

${ }^{2}$ Department of medical record management, Affiliated Hospital of Hebei University, Baoding 071000, Hebei Province, China

Abstract: Geraniol is an acyclic monoterpenoid compound, which exists widely in aromatic plants. Geraniol has antibacterial and anti-inflammatory effects. Recently, it has been found that geraniol has a strong effect on improving immune function and anti-tumor. Many experimental evidences support that geraniol has a good effect on the treatment or prevention of different types of tumors, such as breast cancer, lung cancer, liver cancer, pancreatic cancer, colon cancer, prostate cancer, etc. it also has a synergistic anti-cancer effect with many anticancer drugs, revealing the mechanism of its more complex anti-tumor pharmacological action System. In this review, we summarized a variety of anticancer signaling pathways and targets. Geraniol is considered to be a safe, effective and promising multi-target anti-cancer drug, which is expected to become an important force in the anti-cancer of traditional Chinese medicine.

Key words: Geraniol; Tumor; Genes; Treatment

Publication date: January, 2021

Publication online: 31 January, 2021

"Corresponding author: Zhi Hao, xishuai761011@ sina.com

\section{Introduction}

Geraniol is an acyclic monoterpenoid with the molecular formula C10H18O.As a plant extract, it was first isolated from palmarosa. At present, it has a wide range of sources, such as Geranium of Geranium of yak family, Rutin of citronella of Gramineae, flowers and palms of Rosa of Rosaceae; Geraniol can also be extracted from the pupae of some animals, such as male Tetranychus urticae ${ }^{[1]}$. Geraniol is light yellow, insoluble in water, easily soluble in gasoline, alcohol, benzene and other organic solvents, with sweet rose flavor, so it is often used to make essential oil and spices. Due to its large demand, its preparation mostly comes from the industrial production with laurene as raw material ${ }^{[2]}$.

Geraniol has antibacterial, anthelmintic and antiinflammatory effects; Recently, it has been found that it can improve the immune function and antitumor effect. Geraniol exerts its pharmacological and biological effects largely due to its strong antioxidant activity $^{[3,4]}$.

\section{Antioxidant activity}

Free radicals can oxidize cell molecules and eventually produce molecular changes related to aging, tumor, atherosclerosis, diabetes and asthma ${ }^{[5]}$. Recently, studies have confirmed that geraniol has antioxidant and free radical scavenging effects. Choi et $\mathrm{al}^{[6]}$ Evaluated the free radical scavenging ability of geraniol in vitro, and the scavenging rate was $87.7 \%$, which was equivalent to that of $235.9 \mathrm{mg}$ watersoluble vitamin E; Tiwari ${ }^{[7]}$ showed that in evaluating the antioxidant capacity of geraniol by inhibiting rat alveolar macrophages, geraniol significantly increased the cell survival rate, the activity of SOD increased by more than $45 \%$, the content of GSH increased by more than $120 \%$, and the accumulation of mitochondrial membrane potential increased significantly. Geraniol can also significantly reduce lipid peroxidation, inhibit the release of nitric oxide (no) and the proliferation of reactive oxygen species $(\mathrm{ROS})^{[8]}$. These results suggest that geraniol as a drug to enhance immunity and anti-tumor, strong antioxidant reaction is a key point. 


\section{Antitumor effect}

In vivo and in vitro experiments have confirmed that geraniol has antitumor effect in many human cancer models ${ }^{[9]}$. It has been found to inhibit the survival and growth of a variety of cancer cells. However, the anti-tumor mechanism of geraniol has not been fully understood, many scholars at home and abroad have done a lot of research work, trying to clarify it from the gene level.

$\mathrm{Yu}$ et $\mathrm{al}^{[10]}$ Found that geraniol was used to feed SD rats two weeks before 7,12 - (a) anthracene dimethylbenzo induced breast cancer. After 22 weeks, SD rats showed the ability to inhibit the diversity of breast cancer, which could reduce the risk of breast cancer by $45 \%$. The action site of isoprene was considered as the HMGR target of geraniol inhibiting sterol resistance. Cho ${ }^{[11]}$ suggested that geraniol could inhibit the growth of human breast cancer MCF7 cells by inducing breast cancer cells to arrest in G1 phase. Cyclins D1, a, e and CDK4 decreased, but p27 cells increased. Moreover, geraniol did not affect the growth of normal breast epithelial cells $\mathrm{f}-10 \mathrm{f}$, indicating that its activity was tumor specific. Although geraniol inhibited the activity of 3-hydroxy-3-methylglutaryl coenzyme A (HMG $\mathrm{CoA}$ ) reductase, geraniol induced cell cycle arrest could not rescue the product of HMG CoA reductase by supplementing mevalonate. These results suggest that the antiproliferative effect of geraniol on MCF7 cells is not related to the decrease of $\mathrm{HMG} \mathrm{CoA}$ reductase activity or the limitation of mevalonate level, but related to other molecular mechanisms. Duncan $^{[12]}$ found that geraniol can inhibit the proliferation of breast cancer cell MCF-7, inhibit cell cycle progression and reduce the activity of CDK2. Geraniol significantly inhibited the proliferation and DNA synthesis of MCF-7 cells, blocked the distribution of cell cycle in $\mathrm{S}$ phase, decreased the expression of cyclin D 1 and CDK 2, induced apoptosis and increased the expression of p-p38.Low dose geraniol $(<50 \mu \mathrm{mol} / \mathrm{L})$ significantly increased QR activity, decreased cdk-2 protein expression and decreased PGE2 release. In addition, geraniol also significantly reduced the expression of cdk-2 protein in rat breast cancer tissues. Therefore, it is speculated that geraniol may inhibit the proliferation of breast cancer cells by down regulating the activity of cdk-2

Galle et al ${ }^{[13]}$ Reported the effect of geraniol on the proliferation of human lung cancer cell line A 549 in nude mice, and observed the effect of geraniol added at 25,50 and $75 \mathrm{mmol} / \mathrm{kg}$ on the proliferation of a 549 cells, which showed that geraniol had a dose and time-dependent growth inhibition effect on a 549 cells, inhibited tumor growth in vivo, and induced apoptosis; Further experiments in vivo showed that geraniol reduced 3-hydroxymethylglutaryl coenzyme-A reductase, which is the rate limiting enzyme of cholesterol synthesis. With the formation of cholesterol and cholesteremia, geraniol reduced the number of membrane bound Ras protein.

\section{Conclusion}

Geraniol has no obvious effect on normal mouse hepatocytes, but significantly inhibits mevalonate pathway, which is closely related to the proliferation and apoptosis of A549 tumor cells. Geraniol has obvious anti-tumor activity. Gysin ${ }^{[14]}$ and other studies showed that geraniol reduced the expression level of Ras in A549 cell membrane, and there was no corresponding change in the protein number of Ras in normal mice. This may be due to the inhibition of mevalonate pathway induced by geraniol, resulting in the decrease of isoprene of Ras.

Burke $^{[15]}$ and others studied the mechanism of geraniol's anti pancreatic cancer effect. They observed that geraniol could increase the expression of Pro apoptotic protein Bak and further induce apoptosis of pancreatic cancer cells in vitro; Moreover, geraniol can completely inhibit the growth of PC-1 pancreatic cancer cells and significantly inhibit the growth of human pancreatic cancer suspension cells Mia PaCa-2 by feeding Syrian golden hamsters with 20 $\mathrm{g} / \mathrm{kg}$ geraniol solution. Wiseman ${ }^{[16]}$ reported that geraniol can induce the production of cell cycle kinase inhibitors p21cipl and p27kip1 in human pancreatic cancer cells, resulting in the decrease of CDK2 activity and the expression of tumor cell cycle related downstream proteins, which eventually blocks the cell cycle in G0 / G1 phase, thus inhibiting the growth and withering of pancreatic cancer cells. Jin Xiaoxin $^{[17]}$ and other researchers found that geraniol has obvious inhibitory effect on transplanted tumor of human pancreatic cancer cell BXPC-3 in nude mice. Compared with gemcitabine group, geraniol has similar anti-tumor effect. To study its anticancer mechanism, the following factors may exist: 1 . 
Inhibit the activity of $\mathrm{NF}-\kappa \mathrm{B}$ and the expression of survivin, livin and $\mathrm{Bcl}-2$, and up regulate the expression of Bax;2. Geraniol intervention can make cytochrome c flow into the cytoplasm of pancreatic cancer cells from mitochondria, activate caspase, change the mitochondrial structure of pancreatic cancer cells, and cause apoptosis of pancreatic cancer cells;3. Geraniol can also inhibit the expression of MMP2, MMP9 and NF - $\kappa \mathrm{B}$, promote the expression of TIMP2, and inhibit the metastasis and invasion of pancreatic cancer cells.

Geraniol can also inhibit the proliferation of human hepatoma cell line HepG2. Fu Xueyan ${ }^{[18]}$ reported that geraniol at different concentrations can promote the apoptosis of HepG2 cells, especially in combination with p-propylbenzaldehyde, with more obvious effect, but no obvious damage to normal liver cells. Xu Hui ${ }^{[19]}$ reported that geraniol could significantly inhibit the growth of human hepatoma cell line Huh7. The results of flow cytometry showed that geraniol could significantly reduce the growth of human hepatoma cell line Huh7 However, the mRNA and protein expression of TGF-b1 and Smad2 were significantly inhibited by geraniol in a concentration time dependent manner. The mechanism may be that geraniol can inhibit the growth of hepatoma cells by blocking the TGF-b1 / Smad2 signaling pathway. $\mathrm{Polo}^{[20]}$ reported that geraniol can significantly inhibit the apoptosis of Hep G2 cells, and the effect is more obvious when combined with simvastatin. The mechanism may be related to the effect on the metabolism of mevalonate in Hep G2 cells. Ong ${ }^{[21]}$ and other studies have confirmed that geraniol can inhibit cell proliferation and promote DNA damage of hepatoma cells at the initial stage of RH model, and increase the activity of glutathione-S-transferase in hepatoblasts to increase the positive apoptosis of precancerous lesions.

Carnesecchi ${ }^{[22]}$ studied the effect of geraniol on the growth of human colon cancer cell line Caco-2. Geraniol $(400 \mu \mathrm{m})$ inhibited the growth of Caco-2 cells by $70 \%$, arrested the tumor cells in S phase of cell cycle, and inhibited the synthesis of DNA. No normal cytotoxicity or apoptosis was found. This may be related to the decrease of ornithine decarboxylase activity caused by geraniol. Ornithine decarboxylase is the key enzyme of DNA synthesis and plays a promoting role in the process of tumor growth. Geraniol can also activate the intracellular catabolism of polyamines, which is mainly manifested in the enhancement of polyamine acetylation. These results suggest that polyamine metabolism may be the target of geraniol's antiproliferative effect. Carnesecchi ${ }^{[23]}$ also studied the effect of geraniol on the expression of thymidylate synthase and thymidine kinase in colon cancer cells, which are related to the cytotoxicity of 5-fluorouracil.The antitumor effects of geraniol and 5-fluorouracil on tc-118 human tumor in Swiss mice were also evaluated. Geraniol at 150 $\mu \mathrm{m}$ has been shown to reduce thymidine kinase and thymidylate synthase expression in tc-118 cells of Swiss mice. In nude mice, the tumor volume could be reduced by $26 \%$ by using geraniol $150 \mu \mathrm{m}$ alone, and $53 \%$ by using 5 -fluorouracil $20 \mathrm{mg} / \mathrm{kg}$ in combination. 5-fluorouracil alone had no significant effect, indicating that geraniol and 5-fluorouracil have synergistic anticancer effect. The possible mechanism is that geraniol increases the permeability of cell membrane and the ability of colon cancer cells to absorb 5-fluorouracil; The improvement of cell membrane permeability also leads to changes in cell resting potential and membrane polarity, further changes in the ability of membrane binding proteins, and changes in intracellular signaling pathways ${ }^{[24,25]}$. Mans ${ }^{[26]}$ and other scholars also confirmed that thymidine kinase and thymidylate synthase are related to the antitumor activity of 5-fluorouracil to a certain extent, and reducing the activity of these two enzymes can improve the anti-tumor activity of 5-fluorouracil.

Geraniol also shows good anti-cancer effect in urogenital system tumors. Ahmad et $\mathrm{al}^{[27]}$ found that after iron triacetate induced renal oxidative stress and cell canceration in Wistar rats, inflammatory reaction and cell proliferation would occur in rats. At this time, geraniol (100-200mg / kg) gavage for 16 weeks could significantly inhibit its oxidative stress and inflammatory reaction, and the effect was remarkable. The proliferation of tumor cells was significantly inhibited. The results of histopathology and immunohistochemistry showed that the LDH, creatinine and urea nitrogen water in geraniol group were significantly lower than those in iron triacetate group. Geraniol inhibited the mRNA expression of apoptosis factors KIM-1, NF KB, PCNA and p53 in a dose-dependent manner, and significantly increased the levels of glutathione GSH and apoptotic protease in kidney, It can effectively improve the renal injury caused by iron triacetate in animal 
model. Apoptosis and autophagy are closely related to tumor. Studies have found that geraniol can induce apoptosis and autophagy of PC-3 cells in vitro, and inhibit the proliferation of tumor cells. The levels of Caspase-3, LC3 and LC3 - II in geraniol group were significantly increased ${ }^{[28]}$. Shan et $\mathrm{al}^{[29]}$ Observed that geraniol also had a good inhibitory effect on endometrial cancer in Wistar rats. Western blotting method was used to detect K-ras, MAPK, PI3K, Wnt / $\beta$ - Catenin gene, TGF - $\beta$ and PCNA, PTEN, progesterone receptor and E-cadherin in endometrial cancer tissues and control group. Oral administration of geraniol can reverse the normal development of mRNA expression. It can induce the up regulation of PCNA expression and down regulation of PTEN, progesterone receptor and E-cadherin expression in rats, which can significantly reverse the protein expression pattern. This result provides strong evidence for geraniol to affect the regulation of MAPK pathway and Wnt signaling pathway in the prevention of endometrial cancer in rats.

Geraniol also showed a strong anti-cancer effect in other rare malignant tumors, such as other malignant melanoma and oral cancer. Madankumar et al ${ }^{[30]}$ showed that geraniol (200mg / kg, 3 times a week) could significantly inhibit the proliferation of oral squamous cell carcinoma induced by $4 \mathrm{NQO}$ in rats after intragastric administration for 20 weeks. The apoptosis could be observed by histological and electron microscopic studies. The mechanism may be that geraniol can regulate the activities of phase I / II coupling metabolic enzymes in tongue and liver. Geraniol can also inhibit the growth of melanoma cells. Yu et al ${ }^{[31]}$ reported the dose-dependent effect of geraniol on the growth of B16 melanoma in mice.5 $\mathrm{mmol} / \mathrm{kg}$ geraniol could significantly inhibit the growth of melanoma cells $(P<0.02)$. The mechanism might be that geraniol could inhibit the activity of 3-hydroxy-3-methylglutaryl coenzyme A (HMG $\mathrm{CoA}$ ) reductase, so as to inhibit the growth of melanoma cells.

In a word, geraniol exists widely in many animals and plants, and has been successfully synthesized in batches. Many studies have confirmed its extensive drug activity, especially its strong anti-cancer effect on many tumors. It is likely to become an important force in the anti-cancer of traditional Chinese medicine, but its specific pharmacological mechanism has not been fully elucidated. This review emphasizes the role of geraniol in many aspects Therefore, geraniol can be used to develop multi-target therapy for better clinical service.

\section{References}

[1] Nanxia F, Zhi-Ling Yang, Yannick Pauchet, et al. A cytochrome P450 from the mustard leaf beetles hydroxylates geraniol, a key step in iridoid biosynthesis[J]. Insect Biochemistry and Molecular Biology, 2019, 113(4): 1302-12.

[2] Chacón M G, Marriott A, Kendrick E G, et al. Esterification of geraniol as a strategy for increasing product titre and specificity in engineered Escherichia coli[J].Microbial Cell Factories, 2019,18(1): 105 .

[3] Madankumar A, Jayakumar S, Gokuladhas K, et al. Geraniol modulates tongue and hepatic phase I and phase II conjugation activities and maycontribute directly to the chemopreventive activity against experimental oral carcinogenesis[J]. European Journal of Pharmacology, 2013,705(20): 148-155.

[4] Galle M, Crespo R, Kladniew B R, et al. Suppression by geraniol of the growth of A549 human lung adenocarcinoma cells and inhibition of the mevalonate pathway in culture and in vivo: potential use in cancer che-motherapy[J]. Nutrition \& Cancer, 2014,66(5):888-895.

[5] Crespo R, Wei K, Rodenak-Kladniew B, et al. Effect of geraniol on rat cardiomyocytes and its potential use as a cardioprotective natural compound $[J]$. Life Sciences, 2017,172(5):8-12.

[6] Choi H S,Sawamura M. Radical-Scavenging Activities of Citrus Essential Oils and Their Components: Detection Using 1,1-Diphenyl-2-Picrylhydrazyl [J]. Special Publication Royal Society of Chemistry, 2001. 48(7):4156- 4161.

[7] Tiwari M, Kakkar P . Plant derived antioxidants - Geraniol and camphene protect rat alveolar macrophages against t-BHP induced oxidative stress [J]. Toxicology in Vitro, 2009, 23(2):295-301.

[8] Venzon L , Mariano L N, Somensi L B, et al. Essential oil of Cymbopogon citratus (lemongrass) and geraniol, but not citral, promote gastric healing activity in mice[ $\mathrm{J}]$. Biomedicine \&Pharmacotherapy, 2017, 98(4): 118-124.

[9] Rekha K R, Selvakumar G P. Gene expression regulation of $\mathrm{Bcl} 2, \mathrm{Bax}$ and cytochrome-C by geraniol on chronic MPTP/ probenecid induced C57-BL/6 mice model of Parkinson's disease[J]. Chem Biol Interact, 2014, 217(9): 57-66.

[10] Yu SG, Anderson PJ, Elson CE. Efficacy of beta -Ionone in the Chemoprevention of Rat Mammary Carcinogenesis[J]. Journal of Agricultur- al and Food Chemistry, 1995, 43(8):2144-2147.

[11] Cho M, So I, Chun J, et al. The antitumor effects of geraniol: Modulation of cancer hallmark pathways (Review)[J]. 
International Journal of Oncology, 2016,48(2):1772-1782.

[12] Duncan, R. E , Lau, et al. Geraniol and b-ionone inhibit proliferation, cell cycle progression, and cyclin-dependent kinase 2 activity in MCF-7 breast cancer cells independent of effects on HMG-CoA reductase activity [J]. BIOCHEMICAL PHARMACOLOGY, 2004, 68(4): 1739-1747.

[13] Galle M, Crespo R, Kladniew B R, et al. Suppression by geraniol of the growth of A549 human lung adenocarcinoma cells and inhibition of the mevalonate pathway in culture and in vivo: potential use in cancer chemotherapy[J].Nutr Cancer, 2014, 66(5):888-895.

[14] Gysin S, Salt M, Young A, et al. Therapeutic Strategies for Targeting Ras Proteins[J]. Genes Cancer, 2011, 2(3):359-372.

[15] Burke Y D, Ayoubia S, Werner S R, et al. Effects of the isoprenoids perillyl alcohol and farnesol on apoptosis biomarkers in pancreatic cancer chemoprevention[J]. Anticancer Research, 2002,22:3127-3134.

[16] Wiseman D A, Werner S R, Crowell P L. Cell cycle arrest by theisopreno- ids perilly alcohol, geraniol, and farnesol is mediated by p-21 Cip1 and p-27 Kipl in human pancreatic adenocarcinoma cells[J]. The Journal of Pharmacology and Experimental Therapeutics, 2007, 320(5): 1163-1170.

[17] Jin XX. Experimental study of geraniol against pancreatic cancer and its mechanism [D].Central South University, 2013.

[18] Fu XY, Huang YJ, Wang L, et al. Effect of geranyl combined with propylbenzaldehyde on proliferation of HepG2 cells and its mechanism [J]. Chongqing Medical Science, 2019, 48(6): 921-924.

[19] XU H, Deng Y. Effects of geranitol on proliferation and TGF- B1/Smad 2 signaling pathway of human hepatocellular carcinoma cell line HUH7 [J]. Journal of Hunan Normal University: Medical Edition, 2017(6): 12-15.

[20] Polo M P, Crespo R, Bravo M G. Geraniol and simvastatin show a syn-ergistic effect on a human hepatocarcinoma cell line[J]. Cell Bio- chemistry \& Function, 2011, 29(6):452-458.

[21] Ong T P, Heidor R, De C A, et al. Farnesol and geraniol chemopreventive activities during the initial phases of hepatocarcinogenesis involve similar actions on cell proliferation and DNA damage, but distinct actions on apoptosis, plasma cholesterol and HMGCOA reductase[J]. Carcinogenesis, 2006,27(6):1194-1203.

[22] Carnesecchi S, Schneider Y, Ceraline J, et al. Geraniol, a component of plant essential oils, inhibits growth and polyamine biosynthesis in human colon cancer cells[J]. Journal of Pharmacology \& Experimental Therapeutics, 2001, 298(1):197-200.

[23] Carnesecchi S, Bras A R, Bradaia A, et al. Geraniol, a component of plant essential oils, modulates DNA synthesis and potentiates 5-fluorour- acil efficacy on human colon tumor xenografts[J]. Cancer Letters, 2004, 215(1):53-59.

[24] Carnesecchi, S. Geraniol, a component of plant essential oils, sensitizes human colonic cancer cells to 5-Fluorouracil treatment[J]. Journal of Pharmacology \& Experimental Therapeutics, 2002, 301(2):625.

[25] Carnesecchi, S. Perturbation by Geraniol of Cell Membrane Permeabi- lity and Signal Transduction Pathways in Human Colon Cancer Cells[J]. Journal of Pharmacology \& Experimental Therapeutics, 2002, 303(2):711- 715.

[26] Mans D R, Grivicich I, Peters G J , et al. Sequencedependent growth inhibition and DNA damage formation by the irinotecan-5-fluorouracil combination in human colon carcinoma cell lines[J]. European Journal of Cancer, 1999, 35(13):1851-1861.

[27] Ahmad S T , Arjumand W, Seth A, et al. Preclinical renal cancer chemopreven tive efficacy of geraniol by modulation of multiple molecular pathways[J]. Toxicology, 2011, 290(1):69-81.

[28] Cho M, So I, Chun J, et al. The antitumor effects of geraniol: Modulation of cancer hallmark pathways (Review) [J]. International Journal of Oncology, 2016,48(5):1772-82.

[29] Shanmugapriya S , Subramanian P , Kanimozhi S . Geraniol Inhibits Endometrial Carcinoma via Downregulating Oncogenes and Upregulating Tumour Suppressor Genes[J]. Indian Journal of Clinical Biochemistry, 2017, 32(2):1-6.

[30] Madankumar A, Jayakumar S , Gokuladhas K, et al. Geraniol modulates tongue and hepatic phase I and phase II conjugation activities and may contribute directly to the chemopreventive activity against experimental oral carcinogenesis[J]. European Journal of Pharmacology, 2013, 705(1-3): 148-155.

[31] Yu S G, Hildebrandt L A, Elson C E. Geraniol, an inhibitor of mevalonate biosynthesis, suppresses the growth of hepatomas and melanomas transplanted to rats and mice[J]. Journal of Nutrition, 1995, 125(11): 2763-2767. 